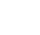

\title{
A telenursing practice for care of people with schizophrenia: Telephone intervention problem solving
}

\author{
Esra Uslu, ${ }^{1}$ @ Kadriye Buldukoğlu, ${ }^{2}$ 는 Lora Humphrey Beebe ${ }^{3}$
}

'Department of Mental Health and Diseases Nursing, Eskişehir Osmangazi University Faculty of Health Sciences, Eskişehir, Turkey

${ }^{2}$ Department of Psychiatric Nursing, Akdeniz University Faculty of Nursing, Antalya, Turkey

${ }^{3}$ Department of Psychiatric Nursing, University of Tennessee College of Nursing, Tennessee, USA

\begin{abstract}
Schizophrenia makes it difficult for people to solve the problems they face in their daily lives. Thus, schizophrenic patients living in a community need special applications to cope with these problems. The Telephone Intervention Problem Solving (TIPS) has been developed by Beebe to serve as a solution for various daily problems of patients with schizophrenia, to offer them coping alternatives, to remind them to use these alternatives and to evaluate the effectiveness of these coping efforts. TIPS, which is conducted with weekly phone calls, is a telenursing practice based on the theory of planned behavior and conducted using problem solving process. Experimental studies conducted on this topic have shown that TIPS extends the time patients with schizophrenia spend in the community, and reduces the number of days spent and applications performed in rehospitalization process. It has also been found that this practice improves the psychiatric medication adherence and reduces the severity of psychiatric symptoms. TIPS was practiced within the context of a doctoral thesis for the first time in Turkey. After the practice, it was concluded that the practice protocol items included in the guideline can be easily used and that it is necessary to rework on only the fourth item. In light of this information, this review article aims to introduce TIPS to psychiatric nurses and to guide them to put TIPS into practice.
\end{abstract}

Keywords: Nurse; schizophrenia; telenursing; telephone.

What is known on this subject?

- Utilization of telemedicine technologies in the care of patients with schizophrenia builds a bridge between the patient and the nurse and yields positive outcomes concerning medication adherence.

What is the contribution of this paper?

- In this study, TIPS was used in the care of patients with schizophrenia for the first time in Turkey.

What is its contribution to the practice?

- TIPS is an appropriate standardized care option that can be transferred to psychiatric nursing interventions.

$\mathrm{O}$ ver 21 million people in the world are living with schizophrenia. The disease damages the thinking, perceptions, emotions, behaviors, and insight of these people. ${ }^{[1]}$ In addition, it adversely affects educational and professional performance, ${ }^{[1]}$ leading to loss of ability and heavy disease burden. [2] However, people affected by schizophrenia can maintain a productive life and be integrated into the community. ${ }^{[3]}$

People with schizophrenia need coping mechanisms to manage the difficulties they face and to protect their health. ${ }^{[4]} \mathrm{At}$ this point, the use of telehealth technologies in the care of people with schizophrenia appears as an opportunity. ${ }^{[5]}$ With telehealth technologies, access to health care services is facilitated, social support can be provided to patients, cost of care decreases, and quality of care increases. ${ }^{[6,7]}$ The use of telehealth technologies in the care of people with schizophrenia also allows these patients to receive positive results from the treatment. ${ }^{[8]}$ These practices also help to bridge the gap between

Address for correspondence: Esra Uslu, Eskişehir Osmangazi Üniv. Sağlık Bilimleri Fak., Ruh Sağlığı ve Hast. Hemşireliği ABD, Eskişehir, Turkey Phone: +90 2222393750 / 1526 E-mail: esra.uslu@ogu.edu.tr ORCID: 0000-0003-0168-2747

Submitted Date: February 16, 2018 Accepted Date: March 18, 2019 Available Online Date: June 26, 2019

${ }^{\circ}$ Copyright 2019 by Journal of Psychiatric Nursing - Available online at www.phdergi.org 
the healthcare professional and patient. ${ }^{[5]}$ Thus, nurses need to use telehealth technologies to ensure continuity of care. ${ }^{[9]}$

The most common telehealth technology used in the care of people with schizophrenia is phone-based practices. ${ }^{[5,10]}$ In this context, periodic telephone interviews provide an economic alternative to encourage therapeutic participation in treatment. ${ }^{[1]]}$ Thus, the telephone has become an effective tool for the delivery of healthcare services. In addition, evidence-based benefits of tele-mental health practices have been determined. ${ }^{[12]}$ Telephone Intervention Problem Solving (TIPS), which is a telenursing intervention conducted with people with schizophrenia using telephone, is documented to contribute to better care of these patients..$^{[4,12-15]}$ Therefore, this review aims to introduce TIPS to psychiatric nurses and to guide them to incorporate TIPS into their practices.

\section{About TIPS}

TIPS is a telenursing practice developed by Lora Humphrey Beebe, ${ }^{[4,16]}$ professor of psychiatric nursing at the University of Tennessee. This practice supports the solution for the daily problems of people with schizophrenia and presents alternatives to cope with these problems. In addition, the TIPS programme reminds clinicians to offer alternative solutions, and to evaluate the effectiveness of these alternatives. TIPS is maintained through weekly phone calls. To implement this intervention, an interview plan of a specific date and time is made with the people with schizophrenia, and the intervention is initiated by TIPS practitioner nurse. The nurse is guided by the TIPS protocol, and the content of each interview is followed up each week with the directions from this guideline. The content of each interview consists of headings associated with the problems experienced by the people with schizophrenia (Table 1).

\section{About TIPS Guideline}

The TIPS guideline is a text consisting of three parts, created

\section{Table 1. TIPS conversation topics}

\section{Topics}

1. Are you taking your medication as prescribed? Have you missed any doses at all?

2. Do you know when your next appointment is scheduled?

3. Have you had any (symptoms) since we last talked? (Symptoms specific to each participant were collected at baseline.)

4. Have you had any cravings for alcohol or other drugs this week that you have found yourselves uncomfortable?

5. How have you been getting along with others this week?

6. Do you have any questions about anything this week?

7. Is there anything else you would like to talk about today?

TIPS: Telephone Intervention Problem Solving. in order to guide psychiatric nurses in the care of people with schizophrenia. ${ }^{[4]}$

First Part defines the positive, negative, and cognitive symptoms seen in schizophrenia. In addition, how cognitive disabilities make everyday problem solving difficult is explained.

Second Part focuses on three specific problem areas, which have been defined by those who have been diagnosed with schizophrenia, including difficulties in treatment adherence, psychiatric symptoms, and individual stress. The problemsolving process is also discussed.

Third Part covers how TIPS can be used in the care of individuals who have been diagnosed with schizophrenia and how people who express their problems about daily living can be answered. The information given in the third part has been standardized by the TIPS protocol.

\section{Sections from the TIPS Guideline}

In this part, only some sections of the TIPS protocol provided in the third part of the guideline will be presented according to the purpose of the article (unclear). The main titles in the protocol, specific verbal expressions and steps that clinicians should follow in implementing TIPS will be defined. The healthcare worker named as "TIPS provider" in the original protocol text is expressed as "nurse" in Turkey, which was faithful to the original of the text.

\section{First TIPS Session}

This session focuses on how talking about treatment and problem solving can help the patients. It is held at the hospital or community mental health center after discharge. Each step in the problem-solving process is explained to the patient with a brief description. In this session, the directive verbal expressions for the TIPS provider are as follows:

\section{Start of the Session}

TIPS provider makes the following statement: "Everyone sometimes has trouble knowing what to do when problems come up. This can be even harder for some people because of their illness. Sometimes people with schizophrenia and similar diseases forget to take medicine, or a doctor's appointment, or don't know what to do when they feel nervous. Problem solving helps you think of what might help when difficulties arise. We will be going through problem solving steps together each week when I call you."

\section{Protocol Item 3}

Ask about symptoms that often precede hospitalization such as anxiety, insomnia, or positive symptoms.

TIPS provider: Have you had any (symptoms) since we last talked? 
Table 2. Sample scenarios

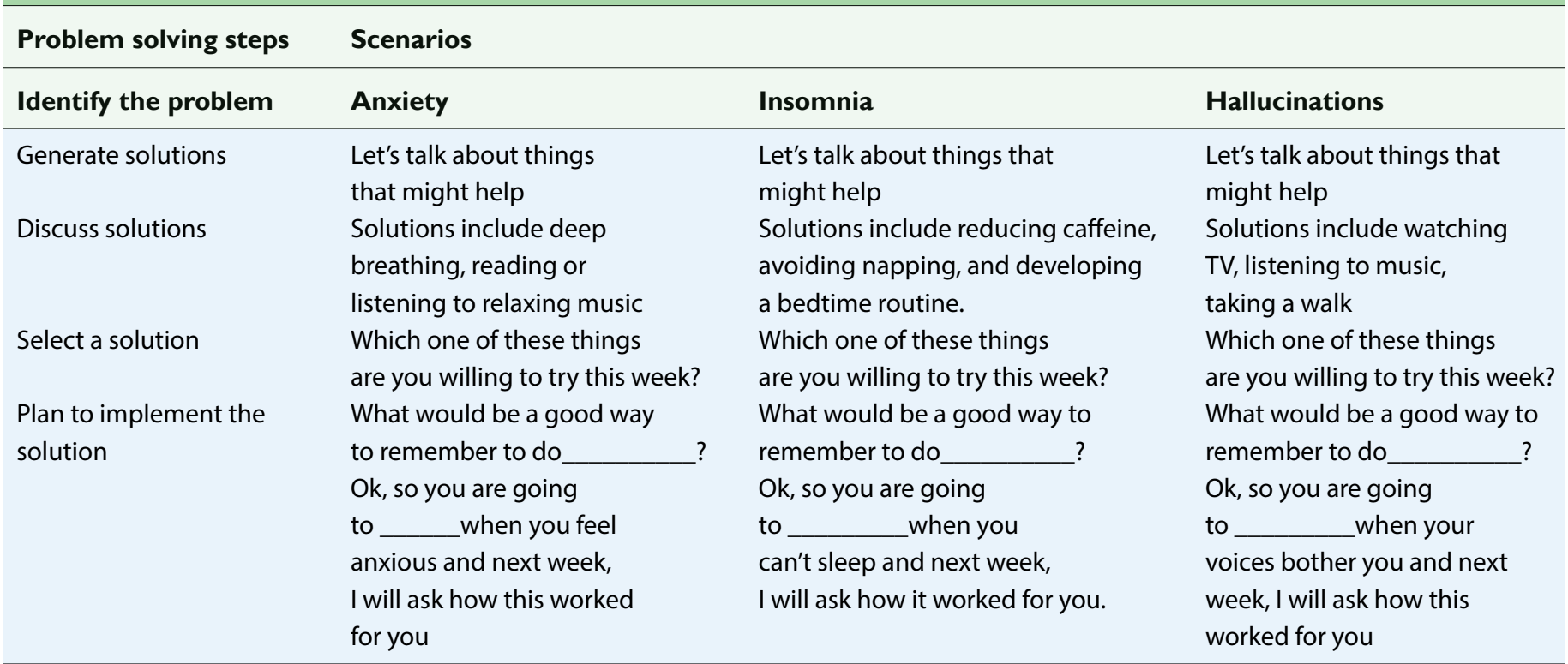

If no, say: "That's good. It is important to let your caregivers know if any symptoms are troubling you."

If yes, ask: "What seems to be the trouble?"

The most common symptoms reported in our prior studies were anxiety, insomnia and hallucinations. Each of these scenarios are presented in the following table (Table 2).

\section{Follow-up Calls}

This part demonstrates how weekly follow-up calls should be maintained.

\section{Frequent and Rare Problems}

This part presents possible problems that the nurse may encounter during TIPS practice, the frequency of these problems, and recommendations that can be effective in the solution of the problem.

\section{Theoretical Basis of TIPS}

"The Theory of Planned Behavior (TPB)", developed by Ajzen, constitutes the basis of TIPS. ${ }^{[15]}$ TPB includes attitude, subjective norms and perceived behavioral control concepts. ${ }^{[17]}$ Attitude in this regard refers to the values and beliefs of an individual in achieving a related behavior. Subjective norm refers to the extent to which significant others (family, caregivers) support the behavior. The perceived behavioral control expresses the difficulty for the individual to exhibit the behavior and the belief of that individual on that topic. ${ }^{[18,19]}$ TIPS is a practice that covers all of these concepts. For example, when the medication adherence of people with schizophrenia is evaluated with TIPS, the attitude represents perceived beliefs and values about the medication adherence. The subjective norm represents at which level families or caregivers encourage the person to adhere to the medication. However, the perceived behavioral control reflects the ease or difficulty of overcoming the obstacles of medication adherence and the level of confidence that one has the ability to do so. Thus, with TIPS practice, the nurse informs the individual about the benefits of medication adherence (attitude), expresses the value of medication adherence, empowers the individual (subjective norm), and solves the problems associated with the obstacles of medication adherence (perceived behavioral control). ${ }^{[15]}$

\section{Research Basis of TIPS}

This section includes the studies examining TIPS. Lora Humphrey Beebe has been involved in all studies as a principal investigator researcher. All studies are randomized controlled trial. The results obtained from these limited studies show that TIPS is a useful telenursing practice for people with schizophrenia. ${ }^{[10]}$

Beebe $^{[13]}$ first assessed the effectiveness of TIPS in the pilot study. The sample of the study consisted of 48 people with schizophrenia. Patients were randomly divided into experimental and control groups. She implemented routine care service for the control group and both routine care service and TIPS for the experimental group as an intervention. In this study, the TIPS practice included interviews which took 12 minutes on average and was carried out on a weekly basis for three months. At the end of three months, the time patients spent in the community, the length of hospital stay, and the frequency of rehospitalizations were assessed. The time that control group spent in the community was 78.25 days, while it was 81.36 days in the experimental group. The length of hospital stay of patients who were rehospitalized in control group 
was found to be 26.13 days, while it was found to be 18.95 days in the experimental group $(\mathrm{t}(4)=-0.724, \mathrm{p}=0.51)$. The frequency of rehospitalization was found to be $23 \%$ in control group, while this ratio was found to be $13 \%$ in the experimental group in this pilot work ( $\mathrm{t}(35)=-0.649, \mathrm{p}=0.52$ ).

Beebe and Tian $^{[4]}$ assessed the effect of conducting a faceto-face interview prior to TIPS on patients in their study. For this purpose, 24 people with schizophrenia were randomly divided into the experimental and control groups. Only TIPS was applied in the control group, and both TIPS and two faceto-face interviews were conducted in the experimental group before discharge. In the study, TIPS practice included interviews which took five minutes on average and were carried out on a weekly basis for six weeks. At the end of the study, when compared to the control group, the duration of phone calls $(F(1,7)=8.49, p=0.02)$ and the number of feeling statement patients expressed increased (Odds ratio $=1.85, p=0.07$ ). The number of one-word response decreased by $50 \%$ in the experimental group (Odds ratio $=0.5355, \mathrm{p}=0.64$ ).

Beebe et al. ${ }^{[16]}$ conducted another study which assessed the effect of TIPS on medication adherence. Accordingly, 29 people with schizophrenia were randomly assigned to experimental and control groups. Treatment was provided to the control group while TIPS was applied to the experimental group. In the study, TIPS practice included interviews which were carried out on a weekly basis for three months. In addition, drugs taken monthly by patients at home were counted to assess medication adherence. As a result, pill count psychiatric medication adherence was found to be $60.1 \%$ on average in the control group, and $80 \%$ on average in the experimental group $(\mathrm{F}(1,20)=5.47, \mathrm{p}=0.0298)$.

Beebe, Smith, and Phillips ${ }^{[14]}$ evaluated the effect of only short text messages, only TIPS, and both short text messages and TIPS on symptom level, and they assessed the medication adherence in people with schizophrenia in their study. For this purpose, patients were randomly divided into three groups. Only short text messages were applied to the first group, while only TIPS was implemented to the second group, and both short text messages and TIPS were applied to the third group. In this study, TIPS practice has included interviews which were carried out on a weekly basis for three months, and short message practice included text messaging once a day for three months. At the end of the study, psychiatric drug adherence score was higher in the group in which both TIPS and daily messaging intervention were used than that in the other two groups. Symptom-level score was found to be 9.2 points lower in the group in which both TIPS and short message intervention were used when compared to the group in which only short message intervention was used, and 5.7 points lower than the group in which only TIPS was used (high scores indicate an increase in symptom level).

Beebe, Smith, and Phillips ${ }^{[15]}$ evaluated the effect of TIPS on medication adherence, medication adherence self-efficacy, and symptom level in their study. The study was conducted with 140 people with schizophrenia. In this study, TIPS was applied weekly for three months. The average length of the interview was three minutes. At the end of three months, the differences were not statistically significant; however, it was found that the symptom level of the patients decreased and the ratio of medication adherence increased. Medication adherence self-efficacy remained stable throughout the threemonth follow-up period.

Recent work on TIPS has been carried out by Beebe. ${ }^{[17]}$ This randomized controlled trial examined the effect of weekly TIPS upon pill count and serum medication levels medication adherence in 119 stable outpatients with schizophrenia for nine months. The results showed no significant differences in psychiatric or nonpsychiatric pill count adherence between groups. However, 29\% of experimental group had serum antipsychotic levels within therapeutic range compared to $19.6 \%$ of control group.

As a result, the duration of TIPS practice varied between six weeks to nine months, and over 350 people with schizophrenia received TIPS. TIPS interviews took less than 10 minutes on average in all studies. None of the participants stated that TIPS practice negatively affected them. On the contrary, TIPS increased the time patients spent in the community, decreased the number of days spent at the hospital in rehospitalizations and frequency of rehospitalizations, ${ }^{[13]}$ increased the psychiatric medication adherence, ${ }^{[14-16]}$ and reduced the severity of psychiatric symptoms..$^{[1,15]}$

\section{TIPS: Turkey}

In Turkey, the effectiveness of TIPS has been examined first and only in the doctoral thesis titled "The Effect of Telephone Intervention Problem Solving on Medication Adherence of Individuals Diagnosed with Schizophrenia" by the first author of the article. ${ }^{[20]}$ This study was carried out using randomized control methods (The effect size was found as $d=2.86$ and the study power 100\%). TIPS was applied to the patients in intervention group every week for two months. The results of the study showed that TIPS contributed to the patients diagnosed with schizophrenia concerning medication adherence to a statistically significant level. In this section, the conspicuous data obtained as a result of TIPS use in Turkey are presented to give an insight to nurses working with people with schizophrenia.

TIPS practice was initiated with 22 patients in the intervention group, but it was completed with the participation of 21 patients since a patient could not be reached after the third week. In the study conducted by Beebe et al., ${ }^{[1]]}$ the ratio of the completed phone calls was found to be $59.3 \%$. The high number of calls completed in the study can be attributed to the conclusion that patients found the practice acceptable and that TIPS can be transferred to psychiatric nursing practices.

In the study, weekly phone calls were made with each patient. Patients completed eight follow-up calls. The interview took 
10 minutes on average. In initial follow-up calls, the duration of calls increased over 10 minutes, while in the last follow-up calls, the duration decreased below 10 minutes. The duration of calls was similar to those in Beebe's studies. It is possible that the shortening of calls' durations over time in the study may be related to the improved problem-solving abilities of patients with schizophrenia.

The study conducted by Beebe and Tian ${ }^{[4]}$ emphasized that it is important to conduct face-to-face interviews with patients before TIPS. A face-to-face interview was carried out during the individualized instruction and TIPS session while pre-test data were collected from all participants, believing that the related thesis study would enhance the effectiveness of TIPS. It is thought that face-to-face interviews prior to TIPS contributed to the positive results achieved at the end of the study.

Beebe stated in the guideline she developed that the patient could not be interviewed during the scheduled time period, which was a frequent problem seen during TIPS practice. In the current study, interviews were not always conducted at the scheduled time period due to patient-related reasons. These problems were often caused by missing the phone call, dead phone battery, forgetting to take the phone, and sleeping at the scheduled time. Therefore, the calls were repeated three times a day, limited to three days in total to contact the patient in accordance with the TIPS protocol. Obtaining the contact information from the patient's relatives before the discharge and communication with the relatives in cases where the patient could not be contacted were effective in the solution of this problem.

The question "Have you ever wanted to use a harmful substance such as alcohol or drugs this week?" in the fourth item of the TIPS protocol was changed after the preliminary application, acquiring the format of the question "Have you needed to use any harmful substances other than your medicines to feel relieved and calm?" when the patients avoided answering or gave short answers. However, during the entire study, participants answered this question as "No". In subsequent studies using this protocol, it will be appropriate to rework or refine the question root.

The TIPS guideline does not provide information about the number of TIPS that is needed to be applied. In the studies conducted by Beebe, the duration of TIPS practice varied from six weeks to nine months, once a week. In the current study, TIPS was applied every week for two months. This time period was effective in reaching the desired results.

In summary, in Turkey example the data obtained after implementing TIPS practice indicated that TIPS can be transferred to psychiatric nursing practices, face-to-face interview to be conducted before TIPS may have a positive effect on the process, the fourth item of the protocol needs to be studied, several calls can be made to reach the participant, and the duration of calls may decrease towards the end of the follow-up.

\section{Conclusion}

This article concludes that TIPS is a practice that supports and utilizes the solution of daily problems experienced by the patients with schizophrenia. It also offers a standardized care option for psychiatric nurses with the use of the protocol. Despite the beneficial effects of TIPS, the limitation of experimental studies examining the effectiveness of TIPS in the care of people with schizophrenia is emphasized in the literature. $[10,15,21]$ These results indicate that researcher nurses are recommended to increase the number of experimental studies evaluating the effectiveness of TIPS and to share the results of these studies with clinicians. In addition, director nurses who are the decision makers of clinics should be informed about the advantages of TIPS applications and their transfer to the clinic. Clinician nurses should also be included in studies that will provide information about the utilization of TIPS.

Conflict of interest: There are no relevant conflicts of interest to disclose.

Peer-review: Externally peer-reviewed.

Authorship contributions: Concept - E.U., K.B., L.H.B.; Design E.U., K.B., L.H.B.; Supervision - E.U., K.B., L.H.B.; Fundings - E.U., K.B.; Materials - E.U., K.B., L.H.B.; Data collection \&/or processing - E.U.; Analysis and/or interpretation - E.U., K.B., L.H.B.; Literature search - E.U., K.B., L.H.B.; Writing - E.U., K.B., L.H.B.; Critical review - E.U., K.B., L.H.B.

\section{References}

1. World Health Organization. Schizophrenia. Available at: http:// www.who.int/mediacentre/factsheets/fs397/en/. Accessed June 24, 2019.

2. Murray CJ, Vos T, Lozano R, Naghavi M, Flaxman AD, Michaud C, et al. Disability-adjusted life years (DALYs) for 291 diseases and injuries in 21 regions, 1990-2010: a systematic analysis for the Global Burden of Disease Study 2010. Lancet 2012;380:2197223.

3. World Health Organization. Mental disorders. Available at: http://www.who.int/mediacentre/factsheets/fs396/en/. Accessed June 24, 2019.

4. Beebe LH, Tian L. TIPS: telephone intervention--problem solving for persons with schizophrenia. Issues Ment Health Nurs 2004;25:317-29.

5. Kasckow J, Felmet K, Appelt C, Thompson R, Rotondi A, Haas G. Telepsychiatry in the assessment and treatment of schizophrenia. Clin Schizophr Relat Psychoses 2014;8:21-27A.

6. Jennett PA, Affleck Hall L, Hailey D, Ohinmaa A, Anderson C, Thomas $\mathrm{R}$, et al. The socio-economic impact of telehealth: a systematic review. J Telemed Telecare 2003;9:311-20.

7. Haley C, O'Callaghan E, Hill S, Mannion N, Donnelly B, Kinsella A, et al. Telepsychiatry and carer education for schizophrenia. Eur Psychiatry 2011;26:302-4.

8. Uslu E, Buldukoğlu K. The Effect of Tele-Nursing Practices to the Schizophrenia Patient Care: A Systematic Review [Article in Turkish]. Turk Psikiyatri Derg 2016;27:47-56. 
9. Nagel DA, Pomerleau SG, Penner JL. Knowing, caring, and telehealth technology: "going the distance" in nursing practice. J Holist Nurs 2013;31:104-12.

10. Uslu E, Buldukoğlu K. The Effect of Tele-Nursing Practices to the Schizophrenia Patient Care: A Systematic Review. [Article in Turkish]. Turk Psikiyatri Derg 2016;27:47-56.

11. Beebe LH. What community living problems do persons with schizophrenia report during periods of stability? Perspect Psychiatr Care 2010;46:48-55.

12. Hailey D, Roine R, Ohinmaa A. The effectiveness of telemental health applications: a review. Can J Psychiatry 2008;53:769-78.

13. Beebe LH. Community nursing support for clients with schizophrenia. Arch Psychiatr Nurs 2001;15:214-22.

14. Beebe L, Smith KD, Phillips C. A comparison of telephone and texting interventions for persons with schizophrenia spectrum disorders. Issues Ment Health Nurs 2014;35:323-9.

15. Beebe LH, Smith K, Phillips C. Effect of a Telephone Intervention Upon Self-Reported MedicationAdherence and Self-Efficacy in Outpatients With Schizophrenia SpectrumDisorders (SSDs). Issues Ment Health Nurs 2016;37:708-14.
16. Beebe LH, Smith K, Crye C, Addonizio C, Strunk DJ, Martin W, et al. Telenursing intervention increases psychiatric medication adherence in schizophrenia outpatients. J Am Psychiatr Nurses Assoc 2008;14:217-24.

17. Beebe LH. Effect of a Telephone Intervention upon Medication Adherence and Related Outcomes in Outpatients with Schizophrenia Spectrum Disorders (SSDs). Med Clin Rev 2017;2:1-6.

18. Ajzen I. The theory of planned behavior. Organic Behavior and Human Decisional Processes 1991;50:179-211.

19. Ajzen I. Perceived Behavioral Control, Self-Efficacy, Locus of Control, and the Theory of Planned Behavior. Journal of Applied Social Psychology 2002;32:665-83.

20. Uslu E. Telefonla Problem Çözme Müdahalesinin Şizofreni Tanili Bireylerin Illaç Tedavisine Uyumuna Etkisi. [Yayınlanmamış doktora tezi] Antalya: Akdeniz Üniversitesi Sağlık Bilimleri Enstitüsü; 2017.

21. Beebe LH, Schwartz. Telephone-based disease management for severe enduring mental illnesses. Home Health Care Manage Pract 2014;26:49-54. 